\title{
Exocentric pointing to opposite targets
}

\author{
Jan J. Koenderink ${ }^{\mathrm{a}, *}$, Andrea J. van Doorn ${ }^{\text {a }}$, Joseph S. Lappin ${ }^{\mathrm{b}}$ \\ ${ }^{a}$ Utrecht University, Faculteit Natuur-en Sterrenkunde, Princetonplein 5, 3584 CC Utrecht, Netherlands \\ b Vanderbilt University, Nashville, Tennessee, USA
}

Received 28 June 2001; received in revised form 1 August 2002; accepted 1 August 2002

\begin{abstract}
We use an exocentric pointing task to study exocentric visual directions to targets that are opposite to a pointer relative to the observer. (The apparent distance between the target and the pointer always exceeded $90^{\circ}$ of visual angle.) All pointing takes place in the horizontal plane at eye height. Observers could not see both target and pointer at a single glance. They had to look back and forth between them, using combinations of eye movements, head turns, twists at the waist and turning on the feet. In the limit of diametrically opposite targets we find that the observers pick either one of two distinct orientations of the pointer as equally "visually correct". Which one results depends on the stance assumed by the observer. The difference between the two equally acceptable pointings is between $5^{\circ}$ and $10^{\circ}$. Such a result is predicted from earlier measurements in the context of a model that describes the geometry of the horizon as a Riemannian space with varying intrinsic curvature. The present results thus fit-perhaps surprisingly — very well in such a picture.

(c) 2002 Elsevier Science B.V. All rights reserved.
\end{abstract}

PsycINFO classification: 2323

Keywords: Visual space; Space perception; Egocenter

\section{Introduction}

Although it appears counterintuitive, "perceptual spaces" are typically differently structured from the corresponding "physical spaces". Nowhere is this more evident than in the space we move in (for most people simply "space"). Yet it is a well established fact that perceptual spatial judgements in the "open loop" situation are typically non-veridical, and systematically so. This appears odd to the rational

\footnotetext{
${ }^{*}$ Corresponding author. Tel.: +31-30-2532808.

E-mail address: j.j.koenderink@phys.uu.nl (J.J. Koenderink).
} 
intellect because one expects that the evolutionary process would have adapted the perceptual spaces to fit the physical ones. Even professional scientists often refuse to believe the evidence from the literature prima facie. Yet the empirical evidence is clear cut for (at least) haptical and optical space. Kappers and Koenderink (1999) found that when blindfolded haptical observers are asked to adjust a rod at one position to be parallel to another rod at another position, they commit errors (referred to physical space) of up to $90^{\circ}$ and more. On removing the blindfold the observers are surprised because their settings look completely wrong, but on closing the eyes the settings feel perfectly acceptable to them. A similar situation applies to optical space. Physically straight lines may look curved, contradicting overwhelming evidence to the rational intellect. In fact, most professionals who routinely deal with spatial issues (architects, pilots, sportsmen, etc.) know about these things or have been forewarned during their early training. Here we talk of perceptual judgements, because it is often the case that sensorimotor acts are metrically dissociated from perceptual judgements. Yet the "perceptual judgements" are typically of the precognitive kind. Spatial relations are perceived "automatically" and cannot be influenced significantly through discursive reasoning.

It is still a moot point whether "perceptual spaces" can be said to exist, or, better, in what sense they can be said to exist. For in any specific psychophysical experiment it is no doubt possible to develop a theory specific for the task and stimulus environment defined by the experimental setting. Then one would have a theory of spatial behavior for every specific task. Doing so would effectively eliminate the need for the notion of a "perceptual space". Of course it would be a scientific major advance if an extensive corpus of such experiments and separate theories could be brought under a single denominator. If successful, then such an overall theory would have a claim to the title "theory of perceptual (substitute the modality) space". This is exactly why the notion of perceptual space is of scientific interest: If it works then one has unified a number of otherwise unrelated threads. Thus one should definitely investigate how far the notion carries one, but not fear to discard it in face of empirical counterevidence.

Historically, human optical space became distinguished from physical space as late as the 19th century. Lotze's (1884) theory of "local sign" can be seen as an early recognition of the topological structure of optical space. Von Helmholtz (1866) and later Mach (1886) and Poincaré (1908) explicitly discuss the psychophysical relations of optical to physical space. The earliest empirical investigations of the geometrical structure of optical space date from the early 20th century (Blumenfeld, 1913; Hillebrand, 1929). Perhaps the first full blown theory of optical space is Luneburg's (1947).

Luneburg's (1947) theory is often construed as an analysis of binocular stereopsis, essentially a geometrical exercise in bicentric perspective. This is not the case though. Although Luneburg certainly refers to binocularity, his theory is much more general and abstract. Luneburg assumes the existence of an optical space completely distinct from physical space. He seeks to arrive at the structure of optical space from a very limited number of elementary assumptions that (ideally) could be doubted by no one on the basis of personal experience. Some assumptions remain implicit, for instance, 
Luneburg assumes the existence of straight lines and planes, and the usual incidence relations between points, lines and planes. Formally this assumes the projective structure of optical space, which is a very strong assumption in the formal (mathematical) sense. Curiously, this assumption remained experimentally untested until very recently (Koenderink, van Doorn, \& Kappers, 2002), since apparently no one doubted its validity. Another (this time explicit) crucial assumption of Luneburg is that of "free mobility of rigid bodies". What this means is that any spatial configuration can be displaced or rotated and still look "the same". It implies the existence of a group of congruences, much like in Euclidian (the default model of physical) space. Free mobility is a very strong constraint since it forces the space to be "homogeneous" (the same everywhere). It has been known since the late 19th century (Bonola, 1955) that there exist only 27 three-dimensional homogeneous spaces (the so-called Cayley-Klein (Klein, 1871, 1872) geometries) of which Euclidian space is one instance. Only three of these are reasonable candidates for optical space (many of the Cayley-Klein geometries have degenerated metrics which are clearly at odds with the simplest observations), namely Euclidian, hyperbolic (or Lobatchevkian) and elliptic space. In elliptic space you would be able to look in the forward direction and see the back of your head (straight lines are closed in elliptic space) which is not borne out by general experience; thus the only remaining contenders are Euclidian and hyperbolic space. Euclidean geometry has zero curvature, hyperbolic geometry is negatively curved, the amount of curvature being arbitrary. During the 1950s and 1960s psychophysicists swallowed Luneburg's theory at face value and the research questions were narrowly focussed upon details of Luneburg's theory (Blank, 1953, 1959) at the cost of a general investigation of the facts. To our view this renders most of the literature on the topic from that period of mere historical interest (with minor exceptions) today: If the general structure of a theory is too far removed from reality, then empirical investigation of its details can only lead to sterile results. (This should not detract from the importance of Luneburg's intellectual achievement of course.) Now that period is over fresh facts (unrelated to any theory) slowly accumulate with result that our understanding of the topic of "visual space" is in a state of flux.

As the empirical evidence accumulated one had to drop the assumption of constant curvature (and with it that of optical space as homogeneous space, see Indow, Inoue, \& Matsushima, 1962a,b, 1963). The notion of optical space remained attractive due to its integrative power though. What is left is optical space as a general Riemannian space of arbitrary (space variant) curvature. This still leaves two categorically different options. The curvature could be a function of the position relative to the observer (e.g., vary as function of the distance to the observer), or it could vary also independent of the observer. In the latter case one would have to assume that the curvature would be determined by what is in the space, i.e., the scene. (In that case optical space would be much like the physical space of Einstein in which the local curvature is determined by the distribution of mass.) Although this latter issue remains unsettled, it is as yet too early (in our opinion) to discard the notion of optical space in the face of empirical evidence.

Unfortunately, the empirical evidence is still remarkably sparse. Almost all of the experiments have been performed in extremely reduced circumstances: Dark room 
with a small number of dim, self-luminous points, fixed skull, in some cases limited eye movements. Data on spatial judgements in realistic (for the daily life situations of human beings) environments are exceedingly rare. And even the results obtained in reduced environments have typically been done in the context of extremely focussed questions (such as "what is the curvature of optical space") rendering the data of very limited general value.

Recently we have introduced a novel method of probing the geometrical structure of human optical space in normal, open environment settings (Koenderink \& van Doorn, 1998; Koenderink, van Doorn, \& Lappin, 2000). In summary, the observer is required to point a pointing device at some location distinct from the vantage point (hence "exocentric" pointing) to a target at another location distinct from the vantage point. This is possible since the pointer is a remote controlled device. The method allows us to sample the directions of geodesics (intrinsically "straight" lines) in human optical space. In general such geodesics differ from the physically straight lines of the space we move in (Blank, 1953, 1959; Blumenfeld, 1913; Hillebrand, 1929; Indow et al., 1962a,b, 1963; Luneburg, 1947). In previous communications (Koenderink \& van Doorn, 1998; Koenderink et al., 2000) we described experiments in which the pointer and the target were at the vertices of equilateral triangles with the observer at the barycenters. In those experiments the pointer and targets were always $120^{\circ}$ apart on the horizon whereas the ratio of their distances was always equal to unity. The data could be described quite well with the model of a Riemannian space of non-constant curvature in which the curvature is a (non-monotonic) function of the distance to the observer. Of course such a description can only be preliminary on account of the limited body of data.

In an attempt to falsify this model we decided to study exocentric pointing in configurations where both the apparent distance between pointer and target varied widely (between about $90^{\circ}$ and almost $180^{\circ}$ ) and the distance ratio varied within a very wide range. This significantly widens the parameter range and the prospective data thus challenge the model. More importantly, the data allow us to check a specific prediction of the model which appears at first blush counterintuitive, namely that pointing to diametrically opposite targets will not be unique. Because the aforementioned model has a curvature singularity at the origin, the (apparently odd ${ }^{1}$ ) prediction is that observers will accept two distinct orientations of the pointer as equally "correct".

Notice that our setting differed in essential respects from the usual laboratory experiment in dark room conditions with reduced response options. In contradistinction, we used free viewing (with eye, head and body rotations permitted) and an intuitively reasonable task in an outdoors (full daylight) setting. Our study may seem

\footnotetext{
${ }^{1}$ The oddity is indeed only apparent. "Pointing" between two points assumedly goes by way of the shortest connection between the points. In most cases that would be the "geodesic" (straight line in terms of the geometry) between the points. But suppose there is some obstacle-say a tree-between the points. Then there exist two equally correct shortest connections, one passing the tree one way, the other passing the tree the other way. A curvature singularity in the space acts essentially like such an obstacle, best circumvented.
} 
less tightly controlled in some ways than a conventional laboratory study, but perhaps it can be generalized to a wider range of full cue conditions. In any case, empirical evidence is needed about spatial perception in full cue conditions. Regrettably, since such studies are comparatively rare (Battro, di Piero Netto, \& Rozestraten, 1976; Battro, Reggini, \& Karts, 1978; Wagner, 1985) our present results are not easily related to the literature.

\section{Methods}

The methods were essentially the same as described in our earlier communications (Koenderink \& van Doorn, 1998; Koenderink et al., 2000). Here we repeat the relevant details.

We used a radio controlled pointer mounted on a tripod (see Fig. 1). The pointer was a cube, pierced with an arrow. The arrow stuck out at both sides. The cube was painted white, the arrow bright orange. The transmitter was a standard Futuba F16 and yielded precise control over the orientation of the pointer. Notice that (due to its design) the orientation of the pointer is easily apparent from monocular cues alone. Targets were bright orange spheres mounted on thin steel rods, themselves mounted on tripods. Sphere diameters were adjusted such that the apparent size of the targets was $20 \pm 4^{\prime}$. The heights of both pointer and targets were adjusted to the eye height of each observer.

Experiments were done in an outside environment. The field was an unkept meadow. Because weeds were growing up to knee and even waist height, it was not often possible to see even approximately where the base of the tripods met the ground.

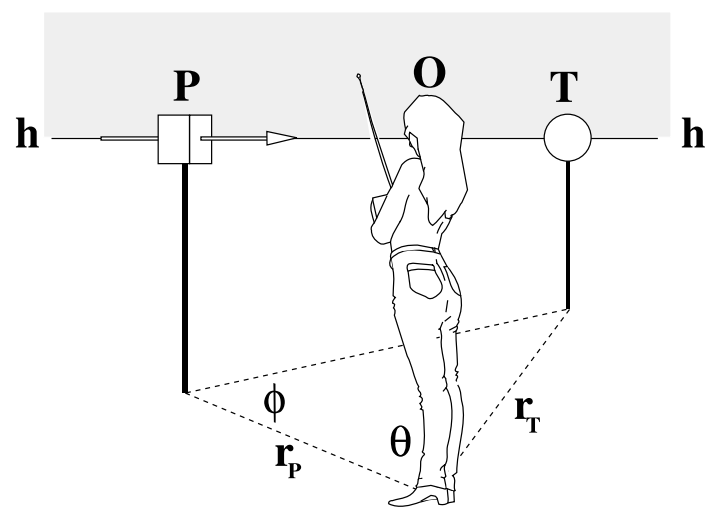

Fig. 1. The exocentric pointing paradigm. The eyes of observer $\mathrm{O}$, target $\mathrm{T}$ and pointer $\mathrm{P}$ are at the same vertical height. Thus the pointer and target appear at the horizon of the observer. The relevant geometry has been drawn on the groundplane (in reality the points on the groundplane directly beneath pointer and target were not visually evident because weeds grew at knee or even waist height). Pointer and target appear at an angle of $\theta$ from each other on the horizon. The veridical pointing angle is $\phi$. The pointing angle is even monocularly evident from the appearance of the pointer. The veridical pointing angle $\phi$ depends on the angle $\theta$ and on the ratio of the distances from the observer to the pointer $\left(r_{\mathrm{P}}\right)$ and the $\operatorname{target}\left(r_{\mathrm{T}}\right)$. 
Since the pointer was at a fixed distance and the targets were adjusted in size, retinal image size could not be an important cue. (Of course the size cue can be used for many details, e.g., weeds, near the tripod.) The area was open, without obvious landmarks, but with some trees and buildings on the horizon. The weather was bright during the course of the experiment. We met with blue sky with less than $1 / 8$ cloud cover. Of course the sun's direction changed appreciably during the course of the experiments.

The observer was instructed to keep the feet within a $40 \mathrm{~cm}$ diameter area and to stand upright at all times. The observer was permitted eye movements, head rotations and torsions at the waist, even changes in placement of the feet (turning in place). The explicit instruction was to point the arrow to the target, i.e., to point it in such a way that someone at the pointer and using it as an aiming device would agree with the setting. Since the observers were not at the pointer (this is exocentric pointing, not aiming), this was not easy, even though most observers found the task a quite "natural" one. At no time were the observers informed of their settings. Three observers (the authors) participated in the experiment. All had normal acuity and binocular stereovision. The observers may be classified as "naïve" with respect to the present task though "experienced in general vision experiments".

Layout of positions was done using conventional geodesic aids (measuring tape and theodolite). Locations are accurate to $10 \mathrm{~cm}$, pointer orientations to about $1^{\circ}$. This turned out to be sufficient given the repeatability of the observer's settings.

\section{Experiment}

We used a configuration based upon a set of collinear target positions. The possible target positions spanned a linear segment of about $25 \mathrm{~m}$ (Fig. 2). Notice that the pointing device and the target were at opposite sides of the observer in the sense that the apparent distance between the target and the pointer always exceeded $90^{\circ}$ of visual angle. Whereas the distance to the pointer was fixed at $2 \mathrm{~m}$, the distance to

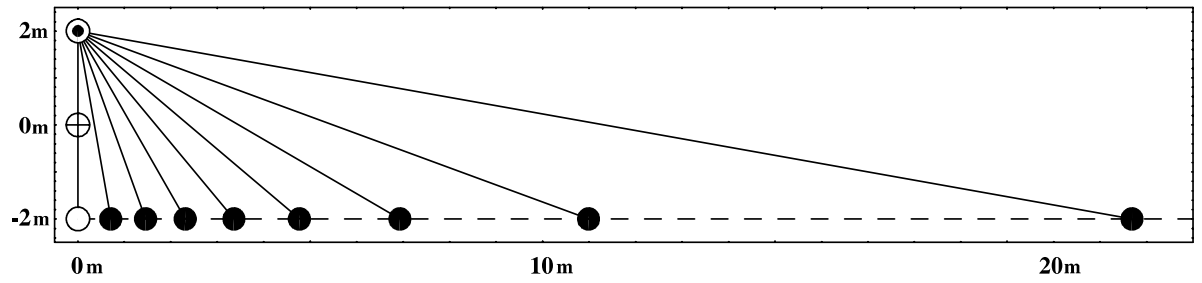

Fig. 2. The configuration for the linear track. Notice that the position of the pointing device is indicated with an open circle with center dot, the position of the observer with a crossed circle. The point on the linear track nearest to the observer is marked with an open circle. The pointer and target would be diametrically opposite in this condition, for this reason this point was never used. The actual target positions used in the experiment are marked with filled circles. This is actually one half of the total configuration because a similar track was laid out towards the left (the observer's position being the same for either track). Thus the total length was about $50 \mathrm{~m}$. 
the target varied greatly. This is important because the veridical pointing angle depends critically upon the ratio of these distances.

Targets could be located at various points on the linear track, we used roughly equal angular (angle $\theta$ in Fig. 1) increments. We always used symmetrical positions (to the left and the right of the observer) and averaged over them. All targets were thus visited twice per session. A total of five sessions brings the total number of settings per target to 10 . In each session we visited the locations in order of decreasing distance. This is almost forced due to time constraints (moving things physically in the field takes time, a session should be done within a day). In a number of random samples we find very similar values as we find in the complete series, thus we have no reason to expect artefacts due to order.

Notice (Fig. 1) that the veridical pointing angles are given by the expression (apply the sine rule of trigonometry for instance)

$$
\tan \phi=\frac{\sin \theta}{\frac{r_{\mathrm{P}}}{r_{\mathrm{T}}}-\cos \theta},
$$

where the angle $\phi$ denotes the pointing angle (taken relative to the visual direction towards the pointer), the angle $\theta$ denotes the visual distance (along the horizon) between the target and the pointer, whereas $r_{\mathrm{P}}$ and $r_{\mathrm{T}}$ denote the distances of the pointer and the target from the observer. Notice that the pointing angle depends only upon the ratio of those distances. Various monocular and binocular cues are available to estimate such ratios, although introspectively this is not what the observers do. The pointing angle $\phi$ can be judged through both binocular and monocular cues. An error analysis suggests that monocular cues may well dominate. (They certainly do at large distances. At closer distances disparity might conceivably play a role, though the pointing angle can easily be judged by monocular vision alone.)

\section{Results}

We used two ways to visualize the raw data. One method is to simply plot the actual against the veridical pointing orientation; this is done in the next section. The other is to notice that a pointer location and orientation with a target location together define a unique parabolic arc. We plot such arcs in order to visualize the pointing behavior of our observers at a single glance (see Fig. 3).

Notice that severe systematic errors do indeed occur. Global similarities and detailed deviations between observers are also apparent.

In Fig. 4 we plot the systematic pointing errors as a function of the visual distance of target and pointer (the angle $\theta$ ). All three observers show qualitatively (and indeed quantitatively) similar behavior. For medium distances $\left(r_{\mathrm{T}}\right)$ all observers exceed the veridical angle sometimes severely $\left(10-15^{\circ}\right)$, for near and far targets they are close to veridical or even show signs of underpointing.

From Eq. (1) one may compute the distance ratio $r_{\mathrm{P}} / r_{\mathrm{T}}$ needed to account for the data. These values differ from the veridical ones and one obvious explanation of this 

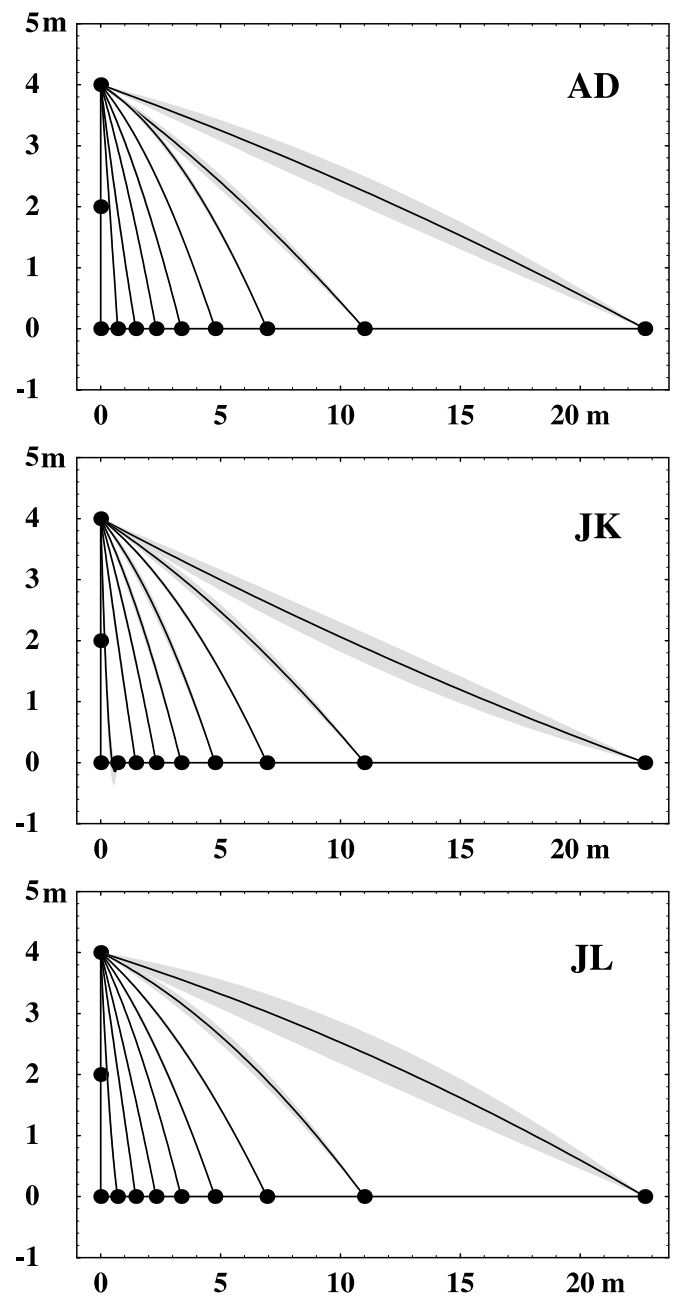

Fig. 3. Overview of the results. The actual pointings have been indicated via parabolic arcs. (Such arcs are uniquely related to the pointings.) The standard deviation in the data is indicated via the gray regions. Notice that these data are averages over symmetrical configurations to the left and the right of the observer. In most cases the pointings were "too far" and consequently the parabolic arcs are convex towards the track on which the targets were placed. In exceptional cases (for instance observer JK at nearest distance) the observer pointed "too close" and the parabolic arc somewhat crosses the track.

pointing behavior may be sought in idiosyncratic "depth estimates". We consider such an explanation unlikely in view of our earlier data: There we covaried $r_{\mathrm{P}}$ and $r_{\mathrm{T}}$ while keeping their ratio at unity, but still we observed significant and systematic pointing errors (Koenderink et al., 2000). Clearly these systematic errors cannot be accounted for by any depth-distance relation, for then the errors would have to be independent of either $r_{\mathrm{P}}$ or $r_{\mathrm{T}}$ taken alone. Whatever the estimated distance ratios $r_{\mathrm{P}} / r_{\mathrm{T}}$ (values that satisfy Eq. (1) for a given value of $\theta$ and the observed value 

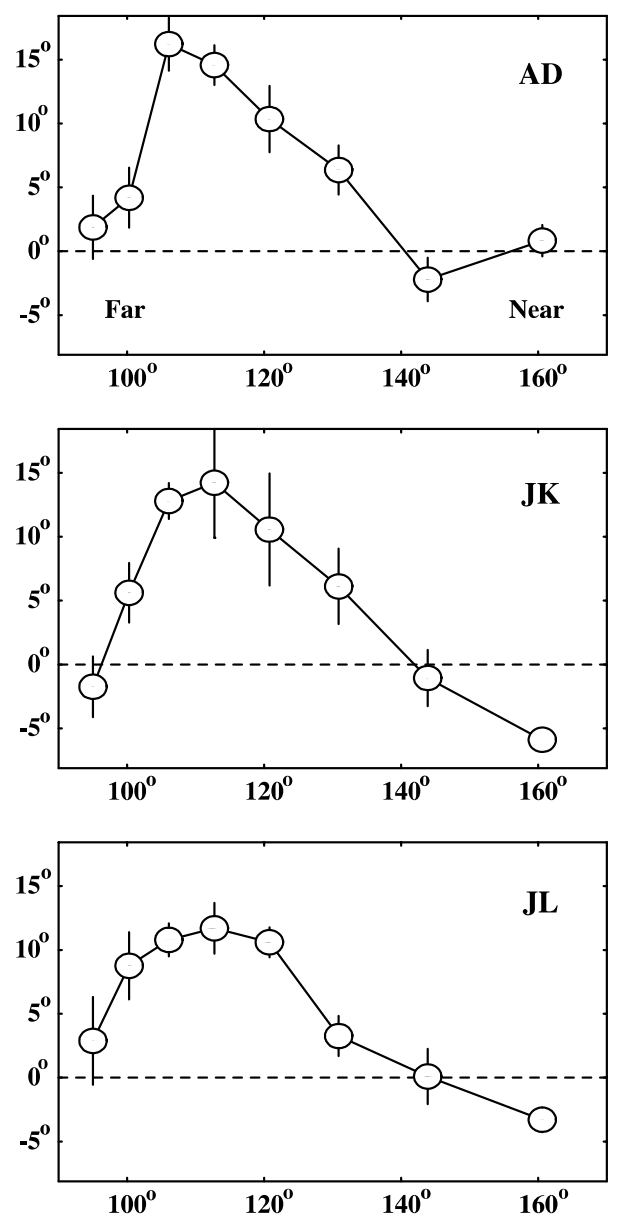

Fig. 4. Systematic errors (in degrees) as a function of the angle $\theta$ (also in degrees). Notice that values of $\theta$ near $180^{\circ}$ indicate cases where the target was placed rather near the observer, whereas values of $\theta$ approaching $90^{\circ}$ indicate cases where the targets were placed relatively far away from the observer. The error bars denote the standard deviations.

of $\phi$ ), might mean, they differed among observers. The estimates were correlated between observers $\left(R^{2} \mathrm{~s}\right.$ are in the $\left.0.68-0.97\right)$, but the ratios for observer JK were more than two times those for $\mathrm{AD}$, and those for JL were about $30 \%$ higher than those for AD. The estimated ratios varied monotonically but non-linearly with the true values.

\section{Pointing to diametrically opposite targets}

It makes little sense to let the observers actually point to targets that are known to be diametrically opposite the pointer because the observers would no doubt adopt 


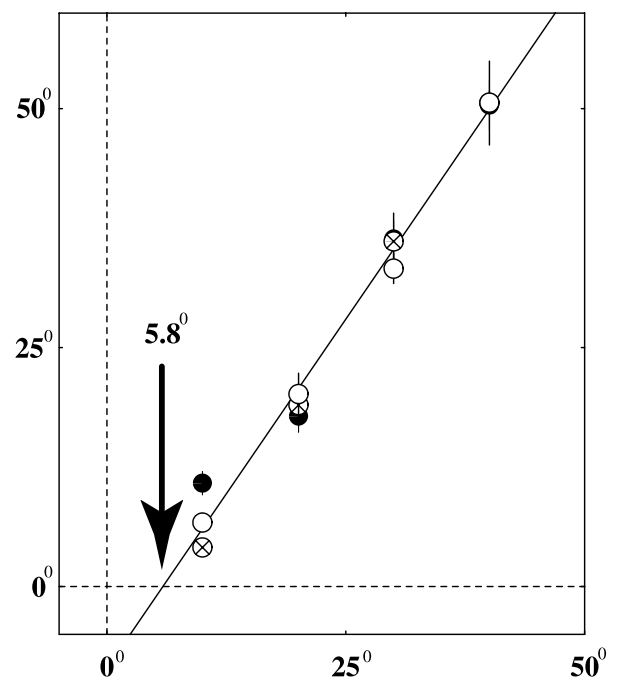

Fig. 5. The obtained pointing angles as a function of the veridical angle, $\phi$. Notice that the regression line misses the origin by about $6^{\circ}$. Data for all observers: $(\bullet) \mathrm{AD},(\otimes) \mathrm{JK},(0) \mathrm{JL}$.

the "winning" strategy of pointing the pointer at themselves. When we tried such an experiment this was invariably what happened. This is not terribly interesting though because the method adopted by the observers in that case is clearly of a singular nature. In such a case it is more revealing to approach the singular situation from either side (because of the symmetry we may average over the two directions) and extrapolate to the limit. This limit may be expected to differ from the "obvious", direct experimental result because it is a limit of the generic case and different from the singular case. ${ }^{2}$

Thus we consider a series of target positions, approaching the diametrically opposite position from two sides. The settings in the contralateral configuration can be interpreted this way. When we plot the pointing angle, the angle $\phi$ as defined in Fig. 1, we would expect the pointing to become veridical for the diametrically opposite position. This would indeed be predicted from almost any theory. The facts are different though. Extrapolating to the diametrical opposite leaves us with a mispointing of $5.8^{\circ}$ (Fig. 5). Notice that Fig. 5 contains the pooled data of all observers; these

\footnotetext{
${ }^{2}$ The following simile might aid the understanding: Consider two locations and a steep hill exactly in the middle. We measure distance by time of travel and we assume that climbing the hill is more expensive than taking a detour. Then there are two generic solutions for the shortest route, one passes the hill on one side, the other passes the hill on the other side. Both solutions will be equally acceptable. There is also a (formal) singular solution, namely the path that passes straight over the hilltop. It is the symmetrical solution (the generic solutions imply a "symmetry breaking") that will be found by many formal algorithms. It is a stationary solution (slightly changing the path will not change the cost linearly), but it is a cost maximum instead of a minimum and thus unstable. When you simulate our experiment for such a situation you will (qualitatively) replicate our empirical result.
} 
data are indeed almost coincident. The predicted value vanishes when the veridical pointing angle reaches a value of $5.8^{\circ}$. Notice that the data are remarkably consistent over the three observers.

Fig. 5 is perhaps misleading in the sense that we approach the origin symmetrically from both sides, whereas only the average has been plotted. It is very important to recognize this because it means that the result must be a branch of a generic symmetrical function and thus linear in a sufficiently small environment of the origin. Thus we have to fit with a straight line through the origin if the limit were indeed the origin. This is obviously far from being the case though. A straight line through the origin fits badly as compared with the offset straight line drawn in the figure. What we have obtained is the typical result of hysteresis: It makes a difference from which side the origin is approached.

Thus we are in the situation that there were two distinct orientations of the pointer that appeared "visually correct" (in the limit) to our observers. Such a state of affairs would occur if the pointing were to follow the geodesics in a space with an intrinsic curvature singularity at the origin. When there is an elliptic excess curvature at the origin this acts as an "attractor" on the geodesics. A simple model is the apex of a cone (see Appendix A).

In our previous studies (Koenderink \& van Doorn, 1998; Koenderink et al., 2000) we found that the geodesic surfaces of the observers had semitop angles of $81-84^{\circ}$. For this surface the predicted pointing error to an antipodal target would have been $0.99-2.2^{\circ}$ (see Appendix A), in contrast to the $5.8^{\circ}$ obtained in the present study. A1though this obtained value seems at first too large, it does not conflict with the previous geodesic surface construction because the prediction of $1-2^{\circ}$ is actually a lower bound. The surface can be bent to make the apex more acute but not flatter; we arrived at a unique interpretation through the constraint that the apex be as flat as possible.

\section{Discussion}

We discuss two topics, one the possible description of the data in terms of depthdistance functions, the other the non-uniqueness of pointing to diametrically opposite targets.

The description of results like this in terms of depth-distance functions is an alternative to the approach via Riemannian metrics. The depth-distance approach is fraught with subtle problems (see Appendix B) and we largely avoid it here. In case a description via depth-distance relations is attempted we can give some constraints on viable depth-distance relations. Informal simulations reveal that the data cannot be fitted with any depth function that displays a purely compressive behavior. This rules out the well known depth functions of the Gilinsky type (Gilinsky, 1951). In order to obtain a fit one needs a depth function that is expansive in the near range and compressive in the far range. One such depth function well known from the literature is that proposed by Luneburg (1947) on theoretical grounds. This function indeed predicts the observed qualitative behavior. However, this function has not enough free parameters to achieve a quantitative fit. 
The prima facie odd prediction from the curved space model that pointing towards a diametrically opposite target will not be unique is-perhaps surprisingly-borne out. Even the magnitude of this odd effect is predicted reasonably well from the previous data (Koenderink et al., 2000). This implies that the observer occurs as a singular point in visual space, in the model the singularity is a concentration of elliptical curvature.

The effect is probably related to the so-called "alleys" (Blumenfeld, 1913; Hillebrand, 1929; Ten Doesschate \& Kylstra, 1956). Indeed, Luneburg's explanation (Luneburg, 1947) of the alleys is very similar to the one given by us for our pointing results. Subjective straight lines are identified with the geodesics in an intrinsically curved space.

Of course the notion that the egocenter is singular is not that surprising in retrospect. It is to be expected that similar behavior can be emulated with different types of models, although the interpretation (here a curvature excess) will differ from case to case.

One may perhaps attempt an explanation different in kind: It may be argued that the egocenter need not necessarily coincide with the nominal location of the observer. An offset of about $20 \mathrm{~cm}\left(5.8^{\circ}\right.$ at $\left.2 \mathrm{~m}\right)$ would account for the offset (see Fig. 6). This displacement, however, should put the egocenter in front of the observer's eyes which seems prima facie unlikely. All of the (scarce) empirical evidence suggests strongly that the egocenter of an observer performing free head movements is located somewhere in the cervical vertebrae. For this reason we discarded the displaced egocenter hypothesis offhand.

However, it seems to us that theories of a non-visual kind (for instance based upon proprioceptive errors) cannot be disregarded very easily. The present experiment certainly does not allow us to decide on that. This is not an issue that can be decided that simply, it will take extensive experimentation.

Intuitively, one may associate this "pointing via curved arcs" with the so-called "subjective curvatures" of objectively straight lines in the visual field as decribed by Hauck (1875) and Von Helmholtz (1866) (see also Pirenne, 1970). The association

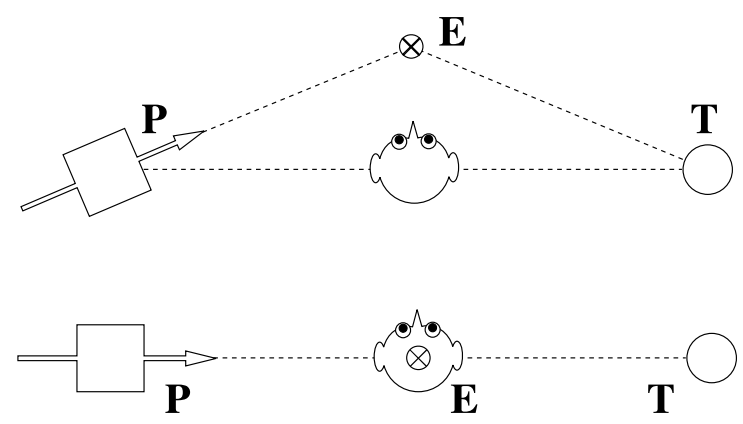

Fig. 6. Pointing to a diametrically opposite target when the egocenter is somewhere in the middle of the head. The observer points the pointer at the egocenter; upper: when the effective egocenter is positioned about $20 \mathrm{~cm}$ in front of the observer that would account for the observed offset in case the observer again points to the effective egocenter. 
is unlikely to apply though, because the subjective curvatures occur only for eccentrically fixated physically present straight objects. Since our observers invariably look back and forth between target and pointer while performing the task, one does not expect this effect to be relevant here.

How does the observer "decide" which of the two equally acceptable pointings to take? A cue is found in the empirical observation that the direction depends on the side from which the target approaches the exact opposite of the pointer position. The observer always points by way of a curved arc that runs in front of the observer. Thus the way the observer faces determines the choice and the observer faces the direction from which the antipode was approached. In the hypothetical situation that there were no "history" and the observer would indeed have to point to the antipode the situation is fully symmetric and we may assume that the choice would be random and determined by the way (picked at random from essentially two possibilities) the observer happens to be facing.

This means that "visual space", when it is taken to include the (unseen) space behind the back, lacks a nice global geometry in the sense that

- there exist lines that meet in two distinct points, and

- it is not the case that any two distinct points determine a unique line.

When the space is limited to only the immediately visible region in front of the observer, then this directional ambiguity does not occur. In many natural situations, however, this directional ambiguity might apply. When one participates in traffic as a pedestrian or as a driver, visually relevant trajectories extend into the space behind as well as in front of the head. The present results may apply to such situations.

Finally, we stress that this paper should be read simply as a report of a surprising but interesting empirical finding. We have tried to place this result in the context of general ideas in the literature of visual space, but we cannot yet offer a general theoretical framework based on only a few facts like these. The theoretical explanation of these results is currently undecided.

\section{Appendix A. The angular separation of geodesics connecting diametrically opposite points}

A simple model for an elliptic singularity at the egocenter is the apex of a cone. On the conical surface (apart from the apex) the intrinsic curvature vanishes; thus the geodesics are simply straight lines. When you cut the surface open along a radius through the target, you see that there are exactly two such straight lines between the pointer and the target because the target appears twice! It is a simple matter to relate the angle between the two directions to the semitop angle of the cone.

Consider a right circular cone of semitop angle $\alpha$ (Fig. 7) and generator length $g$. The radius of the circle at the base of the cone is thus $g \sin \alpha$; hence its circumference is $2 \pi g \sin \alpha$. 


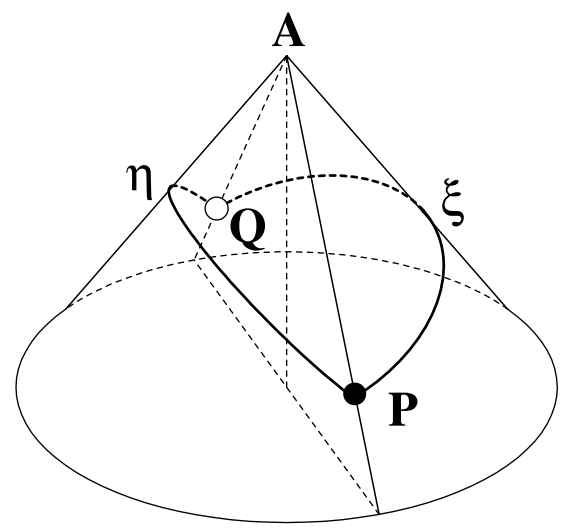

Fig. 7. Points $\mathrm{P}$ and $\mathrm{Q}$ are at opposite sides of a right circular cone with apex $\mathrm{A}$. There exist two distinct shortest connections between $\mathrm{P}$ and $\mathrm{Q}$, these paths $\eta$ and $\xi$ run at either side of the apex. At $\mathrm{P}$ the paths $\eta$ and $\xi$ subtend an angle that is determined by the semitop angle of the cone.

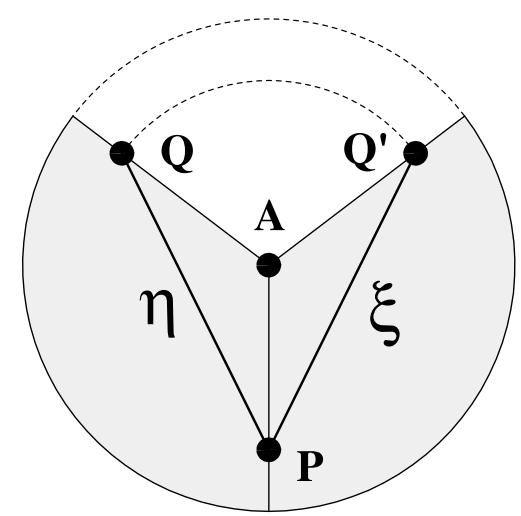

Fig. 8. The same geometry as in the previous figure, except that the cone mantle has been sliced at the generator QA and folded out in the plane. Because the intrinsic curvature of the cone vanishes the cone mantle is intrinsically flat and fits the plane perfectly. The whole plane is not covered though: a sector is missing and the point $\mathrm{Q}$ appears twice $\left(\mathrm{Q}\right.$ and $\left.\mathrm{Q}^{\prime}\right)$. On the plane the shortest connections are simply straight lines, thus the orbits $\eta$ and $\xi$ are the linear segments $\mathrm{PQ}$ and $\mathrm{PQ}^{\prime}$. They subtend a finite angle that is determined by the semitop angle of the cone, namely $\pi(1-\sin \alpha$ ) (see Appendix B), where $\alpha$ denotes the semitop angle.

Now consider a planar fold out of the cone mantle (Fig. 8). It is a circular sector of radius $g$. The sector angle is the length of its arc divided by the radius. Since the length of the sector's arc clearly equals the length of the circumference of the circular base of the cone we obtain a sector angle of $2 \pi \sin \alpha$. Thus the angle of the "gap" is $2 \pi(1-\sin \alpha)$.

From the planar geometry of Fig. 8 one sees immediately that the angle subtended by the geodesics $\xi$ and $\eta$ is one half of the sector angle, thus $\mu=\pi(1-\sin \alpha)$. This is 
the required relation between the angle $\mu$ subtended by geodesics connecting diametrically opposite points and the semitop angle of the cone.

When $\alpha$ is very small the angle $\mu$ is close to $\pi$, and the geodesics leave in opposite directions. This would be the case for a cylindrical surface. When the cone is rather flat the angle $\mu$ will be small. Inserting the values for $\alpha$ of $81^{\circ}$ and $84^{\circ}$ we obtain the values for $\mu$ of $2.22^{\circ}$ and $0.99^{\circ}$ mentioned in the text.

\section{Appendix B. Depth-distance relations}

One may attempt an alternative of the Riemannian space description (a variation of the Luneburg model) in terms of a (fixed) depth-distance relation. The models are equivalent in case the Riemannian space is one of constant curvature, otherwise the predictions will turn out to be different and one may in principle decide empirically between these models. In practice, however, this is not easy because the empirical tolerances are large. At this moment we do not possess sufficient data to make the distinction and we have to leave the problem open in this paper.

Although it seems easy enough to calculate the effects of any (monotonic) depthdistance relation, there are some subtleties to be reckoned with. The problem has to do with the interpretation of the depth-distance relation. In one view one may consider "depth" as judged distance, an eye measure of the separation of things from the observer. In another view one may consider the depth-distance relation as the characterization of the (assumed centrally symmetric) mapping between physical space and the observer's "optical space" or "visual space". Only in the latter interpretation does a relation between the depth description and the Luneburg model make sense. In this view we have to assume that the depth function actually deforms physical space into visual space. Such a deformation also deforms the pointing device and thus changes the apparent pointing angle. Consequently the basic expression (Eq. (1)) has to be corrected for the deformation of the pointer. The analysis is simple enough. We find that the corrected expression also contains the derivative of the depth function (for it is the derivative that describes the local deformation of space in the Luneburg model). We denote the depth-distance relation by $s(r)$, and write $s_{\mathrm{P}}=s\left(r_{\mathrm{P}}\right), s_{\mathrm{T}}=s\left(r_{\mathrm{T}}\right)$. The contraction of space near the pointer in the radial direction is $(\mathrm{d} s / \mathrm{d} r)_{r_{\mathrm{P}}} /\left(s_{\mathrm{P}} / r_{\mathrm{P}}\right)$. Thus we find:

$$
\tan \phi=\frac{\frac{r_{\mathrm{P}}}{s_{\mathrm{P}}}\left(\frac{\mathrm{d} s}{\mathrm{~d} r}\right)_{r_{\mathrm{P}}} \sin \theta}{\frac{s_{\mathrm{P}}}{s_{\mathrm{T}}}-\cos \theta} .
$$

Notice that the condition $r_{\mathrm{P}}=r_{\mathrm{T}}$ does constrain $s_{\mathrm{P}} / s_{\mathrm{T}}$ (has to equal unity) but does not constrain $\left(1 / s_{\mathrm{P}}\right)(\mathrm{d} s / \mathrm{d} r)_{r_{\mathrm{P}}}$. Thus the results from the previous experiments (Koenderink et al., 2000) may still find a possible interpretation in terms of depth-distance functions. Such an interpretation is not possible without the correction, for $r_{\mathrm{P}}=r_{\mathrm{T}}$ implies $s_{\mathrm{P}} / s_{\mathrm{T}}=1$ and thus Eq. (1) shows that the pointing angle $\phi$ should equal the veridical one. When the correction is taken into account (Eq. (A.1)) we find that the tangent of the pointing angle becomes proportional to 
$\left(1 / s_{\mathrm{P}}\right)(\mathrm{d} s / \mathrm{d} r)_{r_{\mathrm{P}}}$. Thus the depth-distance relation does matter in this interpretation and the observations may be used to estimate $\left(1 / s_{\mathrm{P}}\right)(\mathrm{d} s / \mathrm{d} r)_{r_{\mathrm{P}}}$ and thus (by integration) the depth-distance relation itself.

The remarks on depth-distance relations in the discussion apply only in terms of the deformed space interpretation. It is remarkable that the distinction between the depth-distance relation as an eye measure of separation from the observer and the depth-distance relation as a characterization of the relation visual-physical spaces really matters. It is not a mere philosophical issue.

\section{References}

Battro, A. M., di Piero Netto, S., \& Rozestraten, R. J. A. (1976). Riemannian geometries of variable curvature in visual space: visual alleys, horopters, and triangles in big open fields. Perception, 5, 9-23.

Battro, A. M., Reggini, H. C., \& Karts, C. (1978). Perspectives in open spaces: a geometrical application of the Thouless index. Perception, 7, 583-588.

Blank, A. A. (1953). The Luneburg theory of binocular visual space. Journal of the Optical Society of America, 43, 717-727.

Blank, A. A. (1959). The Luneburg theory of binocular perception. In S. Koch (Ed.), Psychology: a study of a science, Vol. I: Sensory, perceptual, and physiological formulations (pp. 395-426). New York: McGraw-Hill.

Blumenfeld, W. (1913). Untersuchungen über die scheinbare Grösse in Sehräume. Zeitschrift für Psychologie, 65, 241-404.

Bonola, R. (1955). Non-Euclidean geometry, and the theory of parallels by Nikolas Lobachevski, with a supplement containing the science of absolute space by John Bolyai. New York: Dover.

Gilinsky, A. S. (1951). Perceived size and distance in visual space. Psychological Review, 58, 460-482.

Hauck, G. (1875). Die subjektive Perspektive und die horizontalen Curvaturen des Dorischen Styls. Stuttgart: Wittwer.

Hillebrand, F. (1929). Lehre von den Gesichtsempfindungen auf Grund hinterlassener Aufzeichnungen. Wien: Verlag von Julius Springer.

Indow, T., Inoue, E., \& Matsushima, K. (1962a). An experimental study of the Luneburg theory of binocular space perception (1). The 3- and 4-point experiments. Japanese Psychological Research, 4, $6-16$.

Indow, T., Inoue, E., \& Matsushima, K. (1962b). An experimental study of the Luneburg theory of binocular space perception (2). The alley experiments. Japanese Psychological Research, 4, 17-24.

Indow, T., Inoue, E., \& Matsushima, K. (1963). An experimental study of the Luneburg theory of binocular space perception (3). The experiments in a spacious field. Japanese Psychological Research, 5, 10-27.

Kappers, A. M. L., \& Koenderink, J. J. (1999). Haptic perception of spatial relations. Perception, 28, 781795.

Klein, F. (1871). Über die sogenannte nichteuclidische Geometrie. Mathematische Annalen Bd., 6, 112145.

Klein, F. (1872). Vergleichende Betrachtungen über neue geometrische Forschungen. Programm zu Eintritt in die philosophische Fakultät und den Senat der Universität zu Erlangen. Erlangen: Deichert.

Koenderink, J. J., \& van Doorn, A. J. (1998). Exocentric pointing. In L. R. Harris \& M. Jenkin (Eds.), Vision and action (pp. 295-313). Cambridge: Cambridge University Press.

Koenderink, J. J., van Doorn, A. J., \& Kappers, A. M. L. (2002). Pappus in optical space. Perception \& Psychophysics, 64(3), 380-391.

Koenderink, J. J., van Doorn, A. J., \& Lappin, J. S. (2000). Direct measurement of the curvature of visual space. Perception, 29, 69-79.

Lotze, H. (1884). Mikrokosmos. Leipzig: Hirzel. 
Luneburg, R. K. (1947). Mathematical analysis of binocular vision. Princeton, New Jersey: Princeton University Press.

Mach, E. (1959). Analysis of sensations and the relation of the physical to the psychical (C. M. Williams, \& S. Waterlow, Trans.). New York: Dover. (Original work published 1886).

Pirenne, M. H. (1970). Optics, painting and photography. Cambridge: Cambridge University Press.

Poincaré, H. (1908). Science et la méthode. Paris: Flammarion.

Ten Doesschate, G., \& Kylstra, J. (1956). The perception of parallels. Ophthalmologica, 131, 61-65.

Von Helmholtz, H. (1866). Handbuch der physiologischen Optik (first ed.). Hamburg and Leipzig: Leopold Voss.

Wagner, M. (1985). The metric of visual space. Perception \& Psychophysics, 38, 483-495. 